\title{
Avaliação de resultados a médio prazo entre artroplastia total de joelho com prótese gênero-específica $x$ prótese convencional*
}

\section{Medium-term Results Evaluation between Gender Specific $x$ Conventional Total Knee Arthroplasty Prostheses}

\begin{abstract}
José Luiz Colleoni $^{1 \oplus}$ Fernando Noel Ribeiro ${ }^{1} \quad$ Alexwell Rodrigues Campos $^{1}$ Djalma de Siqueira Junior ${ }^{1}$ Alysson Guedes Campos ${ }^{1}$ Paulo Augusto Castro Mos $^{1}$

${ }^{1}$ Grupo do Joelho da Disciplina de Ortopedia e Traumatologia do Hospital Estadual Mário Covas, Faculdade de Medicina do ABC (FMABC), Santo André, SP, Brasil

Endereço para correspondência José Luiz Colleoni, Centro de Estudos da Disciplina de Ortopedia e Traumatologia da Faculdade de Medicina do ABC, Rua: Dr. Henrique Calderazzo, 321, Vila Paraíso, Santo André, SP 09190-615, Brasil (e-mail: jcolleoni@uol.com.br).
\end{abstract}

Rev Bras Ortop 2020;55(1):82-87.

\section{Resumo}

\section{Palavras-chave}

- artroplastia do joelho

- feminino

- avaliação de resultados
Objetivo Comparar o resultado funcional pós-operatório das artroplastias totais de joelho (ATJs) com próteses gênero-específicas $x$ convencionais no prazo de 5 anos.

Métodos Estudo retrospectivo com avaliação funcional de 30 pacientes (15 pacientes de cada grupo) utilizando o preenchimento de escores (Knee Injury and Osteoarthritis Outcome Score [KOOS], Western Ontario and McMaster Universities arthritis index [WOMAC] e The Knee Society Clinical Rating System [KSS]) e amplitude de movimento (ADM).

Resultados A diferença encontrada entre os valores de escores e ADM não foram estatisticamente significantes.

Conclusão A prótese gênero-específica apresenta resultados funcionais iguais aos da prótese convencional no prazo de 5 anos de pós-operatorio.
Abstract
Objective To compare the postoperative functional result of total knee arthroplasty (TKA) with gender-specific prosthesis versus TKA with conventional prosthesis in a 5- year period.
Methods Retrospective study with functional evaluation of 30 patients ( 15 patients from each group) using scores (Knee Injury and Osteoarthritis Outcome [KOOS], Western Ontario and McMaster Universities arthritis index [WOMAC] and The Knee Society Clinical Rating System [KSS]) and range of motion (ROM) as methods of assessment.
Keywords
- knee arthroplasty
- female
- outcome assessment significant.
Conclusion The gender-specific prosthesis presents functional results equal to those of conventional prosthesis after 5 years postoperatively.

\footnotetext{
Trabalho realizado no Grupo do Joelho da Disciplina de Ortopedia e Traumatologia do Hospital Estadual Mário Covas, Faculdade de Medicina do ABC (FMABC), Santo André, SP, Brasil.
}

recebido

03 de Julho de 2018

aceito

21 de Agosto de 2018
DOI https://doi.org/

$10.1055 / \mathrm{s}-0039-1697971$. ISSN $0102-3616$.
Copyright $\odot 2020$ by Sociedade Brasileira License terms de Ortopedia e Traumatologia. Published by Thieme Revinter Publicações Ltda, Rio de Janeiro, Brazil 


\section{Introdução}

Diversos fatores podem influenciar no resultado das artroplastias totais de joelho (ATJs), como a amplitude de movimento (ADM) pré-operatória, a técnica cirúrgica adotada, o desenho dos implantes, e a reabilitação pós-operatória.

Historicamente, a ATJ tem obtido sucesso comprovado em diversos estudos de curto e longo prazo na recuperação funcional e no alívio da dor dos pacientes. Atualmente, cerca de $60 \%$ dos pacientes submetidos à ATJ são mulheres, ${ }^{1-3} \mathrm{e}$ muitas discussões têm sido realizadas nas últimas décadas a respeito da influência do gênero nos resultados de curto ou longo prazo. ${ }^{4-7}$

Muitos estudos anatômicos e antropométricos têm demonstrado diferenças morfológicas no fêmur distal das mulheres quando comparado ao dos homens, sendo que o fêmur feminino tem um formato mais trapezoidal, com "offset" ântero-posterior menor e a dimensão dos côndilos mais estreita no eixo médio-lateral. ${ }^{8-10}$

Nos últimos anos, foi introduzido o modelo gênero-específico no mercado de próteses com o intuito de respeitar essas diferenças anatômicas entre os joelhos do homem e da mulher. A diferença desses implantes em relação aos convencionais consiste em um componente femoral com dimensão médio-lateral menor para evitar a proeminência e o contato da prótese com partes moles, o que poderia gerar dor. A espessura do flange femoral anterior também é reduzida para acomodar melhor nos côndilos femininos, objetivando evitar o "overstuffing" do compartimento anterior, e o ângulo da garganta troclear é aumentado em 3 graus, respeitando o ângulo $Q$ aumentado das mulheres. ${ }^{11}$

No entanto, as potenciais vantagens das próteses gêneroespecíficas ainda não foram demonstradas na maioria dos estudos, portanto não há consenso na literatura sobre a necessidade de desenhos de implantes gêneroespecíficos. ${ }^{12-14}$

Dessa forma, o presente trabalho visa realizar uma avaliação comparativa, pós-operatória de médio prazo (5 anos), da satisfação e reabilitação funcional de pacientes do sexo feminino submetidas à ATJ com implantes convencionais e com implantes gênero-específicos.

\section{Métodos}

0 presente estudo foi aprovado pelo comitê de ética em pesquisa da Faculdade de Medicina do ABC (sob o CAAE 51201915.1.0000.5484). O estudo foi realizado de maneira retrospectiva, de abril de 2017 a dezembro de 2017, com 30 pacientes do sexo feminino submetidas à ATJ por gonartrose primária em hospital universitário público.

As pacientes foram divididas em dois grupos com o mesmo número de indivíduos, sendo que no grupo $\mathrm{A}$ foi utilizado o implante gênero-específico Zimmer Gender Solutions Natural-Knee System (Zimmer Biomet, Warsaw, IN, EUA), com modelo desenvolvido para o sexo feminino, e as pacientes do grupo $\mathrm{B}$ foram submetidas à ATJ com implante convencional Exactech Optetrak Logic Primary System (Exactech, Gainesville, FL,EUA).
Todas as cirurgias foram realizadas no período entre janeiro e dezembro de 2012. Os pacientes foram avaliados com 5 anos de pós-operatório.

As cirurgias foram realizadas por cirurgiões diferentes, sendo que em todos os casos, foi adotada a mesma técnica cirúrgica. Todas as próteses foram realizadas com sacrifício do ligamento cruzado posterior (LCP), balanceamento dos espaços flexo-extensão, preservação da patela e da base tibial fixa. Todas as próteses foram fixadas através de cimentação, com utilização de torniquete removido apenas após o fechamento da pele e realização de curativo compressivo. Um dreno aspirativo foi utilizado em todos os joelhos e removido no segundo dia de pós-operatório. Todas as pacientes receberam acompanhamento fisioterápico em que no primeiro dia eram estimuladas a movimentar o joelho e tornozelos, e no segundo dia iniciavam treino de marcha com carga total auxiliada por andador.As pacientes receberam alta no terceiro dia nos casos em que não houve nenhum impedimento de ordem médica.

Todas as 30 pacientes foram avaliadas usando como instrumentos para avaliação dos resultados funcionais os scores: Knee Injury and Osteoarthritis Outcome Score (KOOS), The Knee Society Clinical Rating System (KSS) e Western Ontario and McMaster Universities arthritis index (WOMAC). As pacientes também foram avaliadas quanto ao arco de movimento, além de serem pesadas e medidas na ocasião da avaliação.

\section{Resultados}

A - Tabela 1 contém os dados coletados dos pacientes submetidos à artroplastia com prótese do tipo Exactech. Foram anotados e tabulados os seguintes dados: idade, índice de massa corporal (IMC), KSS, WOMAC, KOOS.

A - Tabela 2 contém os dados coletados dos pacientes submetidos à artroplastia com prótese do tipo gênero-específico (Gender [Zimmer Biomet]). Foram anotados e tabulados dados: Idade, IMC, KSS, WOMAC, KOOS.

A - Tabela 3 contém descrição estatística e comparação entre ambos os grupos estudados para as variáveis de interesse consideradas.

Estes grupos apresentam diferenças ditas estatisticamente não-significantes para todas as variáveis de interesse, ou seja, ambos os grupos são estatisticamente semelhantes (-Figuras 1-4).

\section{Discussão}

Existem diferenças anatômicas bem documentadas entre homens e mulheres em relação ao alinhamento dos membros inferiores e a anatomia do fêmur distal. As mulheres têm um valgo e uma dimensão ântero-posterior do fêmur distal discretamente aumentados, enquanto o diâmetro médiolateral é mais estreito. ${ }^{4,8}$ Esses achados têm levado alguns autores a concluírem que existe a necessidade de desenvolver implantes que se adaptem melhor a essas variações anatômicas. ${ }^{9}$ A premissa fundamental dessa abordagem assume que os resultados das artroplastias totais de joelho realizadas em mulheres são inferiores, e algumas das causas desses resultados piores estão relacionadas a essas 
Tabela 1 Dados dos pacientes submetidos a artroplastias com prótese do tipo Exactech Optetrak (Exactech)

\begin{tabular}{|l|l|l|l|l|l|}
\hline \multicolumn{5}{|l|}{ EXACTECH } \\
\hline Pcts & IDADE & IMC & KSS & WOMAC & KOOS \\
\hline $\mathbf{1}$ & 73 & 29,9 & 74 & 93,9 & 91,1 \\
\hline $\mathbf{2}$ & 71 & 33,5 & 70 & 93,2 & 88,7 \\
\hline $\mathbf{3}$ & 39 & 24,4 & 75 & 93,9 & 91,1 \\
\hline $\mathbf{4}$ & 68 & 31,1 & 74 & 93,2 & 88,7 \\
\hline $\mathbf{5}$ & 74 & 38 & 52 & 92,4 & 87,5 \\
\hline $\mathbf{6}$ & 69 & 37,89 & 77 & 81,8 & 78,5 \\
\hline $\mathbf{7}$ & 79 & 25,3 & 77 & 86,4 & 83,9 \\
\hline $\mathbf{8}$ & 75 & 32,6 & 72 & 87,9 & 85,7 \\
\hline $\mathbf{9}$ & 73 & 33,7 & 77 & 84,1 & 81,5 \\
\hline $\mathbf{1 0}$ & 80 & 36,97 & 62 & 81,9 & 78,6 \\
\hline $\mathbf{1 1}$ & 80 & 34,6 & 64 & 83,3 & 80,4 \\
\hline $\mathbf{1 2}$ & 73 & 28,7 & 74 & 88,6 & 85,1 \\
\hline $\mathbf{1 3}$ & 80 & 25 & 75 & 80,3 & 76,2 \\
\hline $\mathbf{1 4}$ & 86 & 33,3 & 75 & 90,9 & 85,7 \\
\hline $\mathbf{1 5}$ & 78 & 35 & 79 & 88,6 & 85,1 \\
\hline MÉDIA & 73,2 & 31,997 & 71,8 & 88,026 & 84,52 \\
\hline
\end{tabular}

Abbreviations: IMC, índice de massa corporal; KOOS, Knee Injury and Osteoarthritis Outcome Score; KSS, The Knee Society Clinical Rating System; Pcts, pacientes, WOMAC, Western Ontario and McMaster Universities arthritis index.

Tabela 2 Dados dos pacientes submetidos a artroplastias com prótese do tipo Gender Solutions (Zimmer Biomet)

\begin{tabular}{|l|l|l|l|l|l|}
\hline \multicolumn{6}{|l|}{ GENDER } \\
\hline Pcts & IDADE & IMC & KSS & WOMAC & KOOS \\
\hline $\mathbf{1}$ & 83 & 32,6 & 70 & 83,3 & 81,5 \\
\hline $\mathbf{2}$ & 79 & 30,4 & 74 & 93,2 & 78,6 \\
\hline $\mathbf{3}$ & 78 & 30 & 75 & 87,9 & 85,7 \\
\hline $\mathbf{4}$ & 73 & 34,13 & 77 & 81,8 & 80,4 \\
\hline $\mathbf{5}$ & 64 & 34,8 & 74 & 92,4 & 88,7 \\
\hline $\mathbf{6}$ & 69 & 29 & 74 & 84,1 & 81,5 \\
\hline $\mathbf{7}$ & 74 & 39,1 & 72 & 92,4 & 85,1 \\
\hline $\mathbf{8}$ & 81 & 33,6 & 79 & 93,2 & 91,1 \\
\hline $\mathbf{9}$ & 68 & 34 & 75 & 93,9 & 91,1 \\
\hline 10 & 75 & 33,2 & 72 & 93,2 & 87,5 \\
\hline 11 & 77 & 28,6 & 72 & 88,6 & 85,7 \\
\hline 12 & 71 & 31,2 & 64 & 83,3 & 81,5 \\
\hline 13 & 74 & 29,5 & 60 & 86,4 & 83,6 \\
\hline 14 & 76 & 33,3 & 72 & 93,2 & 88,7 \\
\hline 15 & ÓBITO (dia 04/04/2014) por AVC & \\
\hline MÉDIA & 74,428 & 32,387 & 72,142 & 89,064 & 85,05 \\
\hline & 57143 & 85714 & 8571 & 28571 & \\
\hline
\end{tabular}

Abbreviations: AVC, acidente vascular cerebral; IMC, índice de massa corporal; KOOS, Knee Injury and Osteoarthritis Outcome Score; KSS, The Knee Society Clinical Rating System; Pcts, pacientes; WOMAC, Western Ontario and McMaster Universities arthritis index. diferenças anatômicas e à necessidade de implantes gêneroespecíficos. $^{15}$

Modelos de implantes com desenho gênero-específico foram desenvolvidos baseados nas diferenças anatômicas existentes na extremidade distal do fêmur, quando se comparam homens e mulheres. ${ }^{5,16-21}$ Alguns estudos têm sido realizados tentando estabelecer a superioridade dos implantes gênero-específicos em relação aos convencionais unisex quando utilizados nos joelhos de mulheres, ${ }^{22}$ mas ainda permanece não esclarecido o quanto as diferenças anatômicas entre o homem e a mulher podem influenciar nos resultados da ATJ. ${ }^{23,24}$

Em nosso estudo não encontramos diferença estatística significante em relação à ADM pós-operatória com a utilização de implantes gênero-específicos. ${ }^{12}$ Song et al. ${ }^{12}$ realizaram um estudo prospectivo com 40 pacientes do sexo feminino submetidos à ATJ bilateral simultaneamente em que em um joelho foi utilizado implante convencional e no outro o implante gênero-específico, sendo que, na avaliação da ADM após 2436 meses de pós-operatório, encontraram resultados semelhantes e estatisticamente não significantes entre os grupos. Esses dados contrastam com a hipótese de que os implantes convencionais causam um preenchimento exagerado no compartimento patelo-femoral, uma vez que a dimensão ânteroposterior do côndilo feminino é menor, podendo levar a uma diminuição do arco de movimento pós-operatório. ${ }^{16,25}$ Apesar da altura reduzida da flange no implante Gender Solutions Natural-Knee (Zimmer Biomet), a ADM pós-operatória foi semelhante quando comparamos os dois grupos.

Dois estudos nível I foram conduzidos na Coreia por Kim et al. ${ }^{20,26} \mathrm{com} 223$ pacientes do sexo feminino submetidas à ATJ bilateral, totalizando 446 ATJs. As pacientes foram randomizadas para determinar qual joelho iria receber um implante gênero-específico ou um implante unisex. Os autores não encontraram diferença em nenhum dos estudos em relação à ADM, satisfação, e nos escores KSS e WOMAC. Johnson et al., ${ }^{11}$ após estudo de metanálise envolvendo 253 estudos, afirmam não haver diferença no pós-operatório entre as ATJs e as artroplastias totais de quadril (ATQs) quando se usam implantes gênero-específicos com relação ao uso de implantes convencionais.

Song et al., ${ }^{12}$ em um estudo prospectivo em que 50 pacientes foram submetidos à ATJ bilateral simultâneas, um joelho com implante convencional unisex e outro com implante gênero-específico, afirmam que não observaram diferenças significativas quando comparados em relação à ADM, escore hospital for special surgery (HSS) e WOMAC. Clarke e Hentz, ${ }^{27}$ em estudo prospectivo semelhante com 46 pacientes submetidos à ATJ bilateral simultaneamente, também concluíram não haver diferença funcional pelo escore HSS entre os dois tipos de implantes no pós-operatório com 2 anos de seguimento.

No entanto, sabemos que nosso estudo apresenta uma série de limitações. A primeira delas é o fato de não termos avaliado as condições pré-operatórias como ADM e escores funcionais, sendo que isso é um conhecido fator de influência nos resultados após ATJ; porém, isso foi minimizado pelo fato de excluirmos pacientes com ADM menor que $90^{\circ}$ e com 
Tabela 3 Aplicação do teste de Mann-Whitney, com o intuito de verificarmos possíveis diferenças entre ambos os grupos estudados para as variáveis de interesse

\begin{tabular}{|c|c|c|c|c|c|c|c|c|c|c|}
\hline Variável & Grupo & $n$ & Média & $\begin{array}{l}\text { Desvio } \\
\text { padrão }\end{array}$ & Mínimo & Máximo & $\begin{array}{l}\text { Percentil } \\
25\end{array}$ & $\begin{array}{l}\text { Percentil } 50 \\
\text { (mediana) }\end{array}$ & $\begin{array}{l}\text { Percentil } \\
75\end{array}$ & Valor de $p$ \\
\hline \multirow[t]{3}{*}{ IDADE } & Exactech & 15 & 73,20 & 10,63 & 39,00 & 86,00 & 71,00 & 74,00 & 80,00 & \multirow[t]{2}{*}{0,948} \\
\hline & Gender & 14 & 74,43 & 5,21 & 64,00 & 83,00 & 70,50 & 74,50 & 78,25 & \\
\hline & Total & 29 & 73,79 & 8,34 & 39,00 & 86,00 & 71,00 & 74,00 & 79,00 & \\
\hline \multirow[t]{3}{*}{ IMC } & Exactech & 15 & 32,00 & 4,52 & 24,40 & 38,00 & 28,70 & 33,30 & 35,00 & \multirow[t]{2}{*}{0,930} \\
\hline & Gender & 14 & 32,39 & 2,84 & 28,60 & 39,10 & 29,88 & 32,90 & 34,03 & \\
\hline & Total & 29 & 32,19 & 3,74 & 24,40 & 39,10 & 29,70 & 33,20 & 34,37 & \\
\hline \multirow[t]{3}{*}{ KSS } & Exactech & 15 & 71,80 & 7,22 & 52,00 & 79,00 & 70,00 & 74,00 & 77,00 & \multirow[t]{2}{*}{0,522} \\
\hline & Gender & 14 & 72,14 & 4,93 & 60,00 & 79,00 & 71,50 & 73,00 & 75,00 & \\
\hline & Total & 29 & 71,97 & 6,12 & 52,00 & 79,00 & 71,00 & 74,00 & 75,00 & \\
\hline \multirow[t]{3}{*}{ WOMAC } & Exactech & 15 & 88,03 & 4,83 & 80,30 & 93,90 & 83,30 & 88,60 & 93,20 & \multirow[t]{2}{*}{0,629} \\
\hline & Gender & 14 & 89,06 & 4,53 & 81,80 & 93,90 & 83,90 & 90,50 & 93,20 & \\
\hline & Total & 29 & 88,53 & 4,63 & 80,30 & 93,90 & 83,70 & 88,60 & 93,20 & \\
\hline \multirow[t]{3}{*}{ KOOS } & Exactech & 15 & 84,52 & 4,64 & 76,20 & 91,10 & 80,40 & 85,10 & 88,70 & \multirow[t]{2}{*}{0,759} \\
\hline & Gender & 14 & 85,05 & 4,02 & 78,60 & 91,10 & 81,50 & 85,40 & 88,70 & \\
\hline & Total & 29 & 84,78 & 4,28 & 76,20 & 91,10 & 81,50 & 85,10 & 88,70 & \\
\hline \multirow{3}{*}{$\begin{array}{l}\text { ADM } \\
\text { (graus) }\end{array}$} & Exactech & 15 & 103,33 & 9,00 & 90,00 & 120,00 & 100,00 & 100,00 & 110,00 & \multirow[t]{2}{*}{0,982} \\
\hline & Gender & 14 & 103,57 & 8,42 & 90,00 & 120,00 & 100,00 & 100,00 & 110,00 & \\
\hline & Total & 29 & 103,45 & 8,57 & 90,00 & 120,00 & 100,00 & 100,00 & 110,00 & \\
\hline
\end{tabular}

Abbreviations: ADM, amplitude de movimento; IMC, índice de massa corporal; KOOS, Knee Injury and Osteoarthritis Outcome Score; KSS, The Knee Society Clinical Rating System; WOMAC, Western Ontario and McMaster Universities arthritis index.

\section{Representação gráfica segundo a variável Idade}

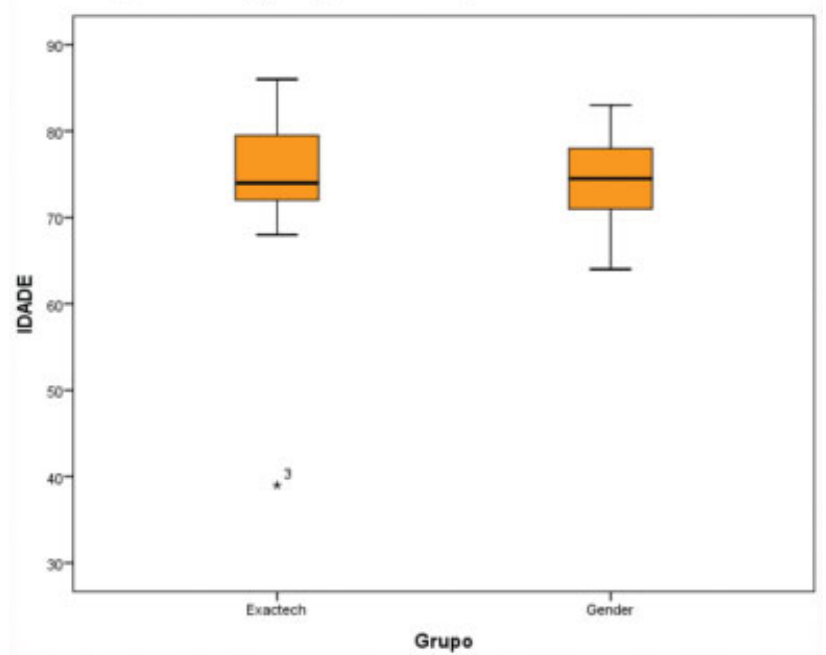

Fig. 1 Representação gráfica segundo a variável idade.

deformidades graves, em que houve necessidade de enxertia óssea ou utilização de implantes de revisão. A segunda é em relação ao médio prazo de seguimento, apenas 5 anos, o que nos impossibilita de obter conclusões de longo prazo a respeito da satisfação; porém, estudos recentes demonstram que esse índices atingem um platô após 1 ano da cirurgia e não sofrem muita alteração após esse período. ${ }^{26,28}$

\section{Representação gráfica segundo a variável IMC}

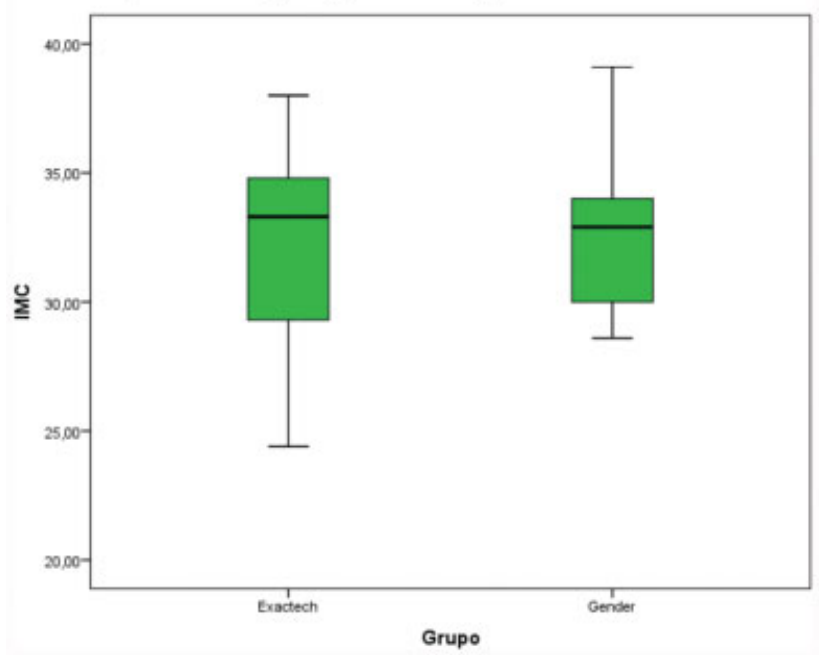

Fig. 2 Representação gráfica segundo a variável índice de massa corporal.

Resumindo, podemos dizer que existem poucos trabalhos na literatura que se referem à análise da eficácia pós-operatória dos implantes gênero-específicos e, em nosso estudo retrospectivo com 30 pacientes do sexo feminino, a aparente superioridade do desenho dos implantes gênero-específicos em relação à melhor adaptação às diferenças anatômicas do joelho feminino não demonstrou resultados clínicos e 
Representação gráfica segundo a variável ADM (Graus)

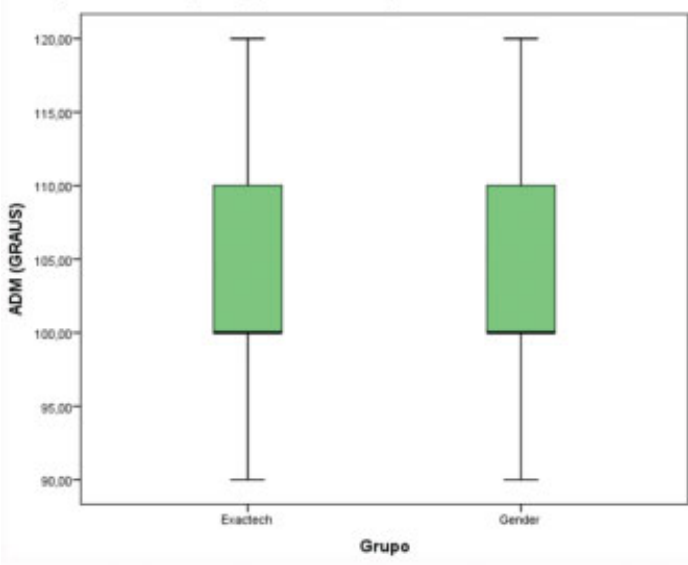

Fig. 3 Representação gráfica segundo a variável amplitude de movimento (graus).

\section{Representação gráfica segundo as variáveis KSS, WOMAC,} KoOS

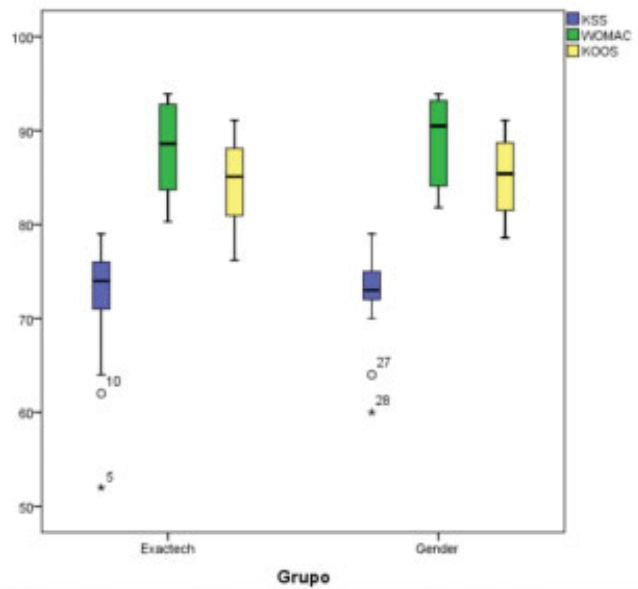

Fig. 4 Representação gráfica segundo as variáveis KSS, WOMAC, KOOS. Abreviaturas: KOOS, Knee Injury and Osteoarthritis Outcome Score; KSS, The Knee Society Clinical Rating System; WOMAC, Western Ontario and McMaster Universities arthritis index.

funcionais superiores quando comparados aos implantes convencionais.

\section{Conclusão}

O presente estudo não demonstrou benefícios clínicos da prótese gênero-específica em pacientes do sexo feminino em um acompanhamento de médio prazo em relação aos implantes convencionais sem distinção de gênero.

Conflito de Interesses

Os autores declaram não haver conflito de interesses.

\section{Referências}

1 Ritter MA, Wing JT, Berend ME, Davis KE, Meding JB. The clinical effect of gender on outcome of total knee arthroplasty. J Arthroplasty 2008;23(03):331-336

2 Font-Rodriguez DE, Scuderi GR, Insall JN. Survivorship of cemented total knee arthroplasty. Clin Orthop Relat Res 1997; ((345):79-86
3 Rand JA, Ilstrup DM. Survivorship analysis of total knee arthroplasty. Cumulative rates of survival of 9200 total knee arthroplasties. J Bone Joint Surg Am 1991;73(03):397-409

4 Hitt K, Shurman JR 2nd, Greene K, et al. Anthropometric measurements of the human knee: correlation to the sizing of current knee arthroplasty systems. J Bone Joint Surg Am 2003;85-A (Suppl 4):115-122

5 Poilvache PL, Insall JN, Scuderi GR, Font-Rodriguez DE. Rotational landmarks and sizing of the distal femur in total knee arthroplasty. Clin Orthop Relat Res 1996; ((331):35-46

6 Livingston LA. The quadriceps angle: a review of the literature. J Orthop Sports Phys Ther 1998;28(02):105-109

7 Woodland LH, Francis RS. Parameters and comparisons of the quadriceps angle of college-aged men and women in the supine and standing positions. Am J Sports Med 1992;20(02): 208-211

8 Chin KR, Dalury DF, Zurakowski D, Scott RD. Intraoperative measurements of male and female distal femurs during primary total knee arthroplasty. J Knee Surg 2002;15(04):213-217

9 Booth RE Jr. Sex and the total knee: gender-sensitive designs. Orthopedics 2006;29(09):836-838

10 Mahfouz M, Booth R Jr, Argenson J, Merkl BC, Abdel Fatah EE, Kuhn MJ. Analysis of variation of adult femora using sex specific statistical atlases. In: Paper presented at: The 7th International Symposium on Computer Methods in Biomechanics and Biomedical Engineering; 2006 Mar 22-25; Côte d'Azur, France.

11 Johnson AJ, Costa CR, Mont MA. Do we need gender-specific total joint arthroplasty? Clin Orthop Relat Res 2011;469(07): $1852-1858$

12 Song EK, Park SJ, Yoon TR, Park KS, Seo HY, Seon JK. Hi-flexion and gender-specific designs fail to provide significant increases in range of motion during cruciate-retaining total knee arthroplasty. J Arthroplasty 2012;27(06):1081-1084

13 Singh H, Mittal V, Nadkarni B, Agarwal S, Gulati D. Gender-specific high-flexion knee prosthesis in Indian women: a prospective randomised study. J Orthop Surg (Hong Kong) 2012;20(02): 153-156

14 Barrett WP. The need for gender-specific prostheses in TKA: does size make a difference? Orthopedics 2006; 29(09, Suppl)S53-S55

15 MacDonald SJ, Charron KD, Bourne RB, Naudie DD, McCalden RW, Rorabeck $\mathrm{CH}$. The John Insall Award: gender-specific total knee replacement: prospectively collected clinical outcomes. Clin Orthop Relat Res 2008;466(11):2612-2616

16 Fehring TK, Odum SM, Hughes J, Springer BD, Beaver WB Jr. Differences between the sexes in the anatomy of the anterior condyle of the knee. J Bone Joint Surg Am 2009;91(10):2335-2341

17 Merchant AC, Arendt EA, Dye SF, et al. The female knee: anatomic variations and the female-specific total knee design. Clin Orthop Relat Res 2008;466(12):3059-3065

18 Gillespie RJ, Levine A, Fitzgerald SJ, et al. Gender differences in the anatomy of the distal femur. J Bone Joint Surg Br 2011;93(03): 357-363

19 Guy SP, Farndon MA, Sidhom S, Al-Lami M, Bennett C, London NJ. Gender differences in distal femoral morphology and the role of gender specific implants in total knee replacement: a prospective clinical study. Knee 2012;19(01):28-31

$20 \mathrm{Kim}$ YH, Choi Y, Kim JS. Comparison of a standard and a genderspecific posterior cruciate-substituting high-flexion knee prosthesis: a prospective, randomized, short-term outcome study. J Bone Joint Surg Am 2010;92(10):1911-1920

21 Greene KA. Gender-specific design in total knee arthroplasty. J Arthroplasty 2007;22(07, Suppl 3):27-31

22 Robertsson O, Dunbar M, Pehrsson T, Knutson K, Lidgren L. Patient satisfaction after knee arthroplasty: a report on 27,372 knees operated on between 1981 and 1995 in Sweden. Acta Orthop Scand 2000;71(03):262-267

23 Khaw FM, Kirk LM, Morris RW, Gregg PJ. A randomised, controlled trial of cemented versus cementless press-fit condylar total knee 
replacement. Ten-year survival analysis. J Bone Joint Surg $\mathrm{Br}$ 2002;84(05):658-666

24 Scuderi GR, Insall JN, Windsor RE, Moran MC. Survivorship of cemented knee replacements. J Bone Joint Surg Br 1989;71(05):798-803

25 Mihalko W, Fishkin Z, Krackow K. Patellofemoral overstuff and its relationship to flexion after total knee arthroplasty. Clin Orthop Relat Res 2006;449(449):283-287

26 Kim YH, Sohn KS, Kim JS. Range of motion of standard and high-flexion posterior stabilized total knee prostheses. A pros- pective, randomized study. J Bone Joint Surg Am 2005;87(07): $1470-1475$

27 Clarke HD, Hentz JG. Restoration of femoral anatomy in TKA with unisex and gender-specific components. Clin Orthop Relat Res 2008;466(11):2711-2716

28 Ritter MA, Berend ME, Harty LD, Davis KE, Meding JB, Keating EM. Predicting range of motion after revision total knee arthroplasty: clustering and log-linear regression analyses. J Arthroplasty 2004;19(03):338-343 